\title{
TENDÊNCIAS RECENTES NOS ELEMENTOS DO CLIMA E SUAS IMPLICAÇÕES NA EVAPOTRANSPIRAÇÃO DA CULTURA DO MILHO EM VIÇOSA - MG
}

\author{
LEONIDAS P. DE ALENCAR ${ }^{1}$, GILBERTO C. SEDIYAMA ${ }^{2}$, \\ EVERARDO C. MANTOVANI ${ }^{3}$, MAURO A. MARTINEZ ${ }^{4}$
}

\begin{abstract}
RESUMO: A mudança nas condições climáticas tem gerado grandes preocupações em nível mundial, sendo que uma das grandes questões, neste contexto, é o aquecimento global. As alterações ocorridas nas condições climáticas podem afetar a demanda de água das plantas, porque a evapotranspiração é afetada por mudanças nos elementos climáticos. O objetivo deste trabalho foi analisar os efeitos das possíveis mudanças dos elementos do clima na evapotranspiração de referência e na evapotranspiração da cultura do milho para a região de Viçosa - MG. Mediante análise de tendência pelo teste de Mann-Kendall e da estatística do teste $t$ da regressão linear, verificou-se o comportamento dos seguintes elementos climáticos: temperaturas máxima e mínima, insolação, velocidade do vento e umidade relativa. Observou-se uma redução na evapotranspiração de referência e da cultura do milho ao longo dos últimos 41 anos, principalmente em razão da redução da insolação e da velocidade do vento. A evapotranspiração de referência apresentou redução da ordem de 4,2 mm por ano.
\end{abstract}

PALAVRAS-CHAVE: evapotranspiração do milho, tendência, mudanças climáticas.

\section{RECENT TRENDS IN THE WEATHER ELEMENTS AND ITS IMPLICATIONS IN THE MAIZE CROP EVAPOTRANSPIRATION IN VIÇOSA-MG, BRAZIL}

\begin{abstract}
The change in weather conditions has caused great concern in the world and the major issue, in this context, is the global warming. The changes in climate may affect the water demand of plants, because the evapotranspiration is affected by changes in climatic elements. The objective of this study was to examine the possible effects of climatological changes in evapotranspiration and reference evapotranspiration of corn in Viçosa - MG, Brazil. Trend analysis by the Mann-Kendall statistic and student t test of the linear regression were used to study the behavior of the following weather elements: maximum and minimum temperatures, sunshine duration, wind speed and relative humidity. It was observed a reduction in the reference evapotranspiration and maize crop evapotranspiration over the recent past 41 years, mainly because of reduced solar radiation and wind speed. The reference evapotranspiration showed reductions of $4.2 \mathrm{~mm}$ per year.
\end{abstract}

KEYWORDS: maize evapotranspiration, trend, climate change.

\section{INTRODUÇÃO}

Um persistente crescimento na quantidade de dióxido de carbono na atmosfera, que pode ser causado pela industrialização e desmatamento, tem sido registrado ao longo das últimas décadas. Esta tendência pode levar a mudanças nas características do clima: temperatura, umidade relativa e precipitação, tanto em níveis globais como regionais (YU et al., 2002).

Atualmente, a mudança nas condições climáticas tem gerado grandes preocupações em nível mundial, sendo que uma das grandes questões do presente século é o aquecimento global.

\footnotetext{
${ }^{1}$ Eng $^{\mathrm{o}}$ Agrícola e Ambiental, Doutorando, Departamento de Engenharia Agrícola, UFV, Viçosa - MG, Fone: (0XX31) 3899.1900, lpaufv@hotmail.com.

${ }^{2}$ Doutor, Prof. titular, Departamento de Engenharia Agrícola UFV/Viçosa - MG, g.sediyama@ufv.br.

${ }^{3}$ Doutor, Prof. titular, Departamento de Engenharia Agrícola UFV/Viçosa - MG, everardo@ufv.br.

${ }^{4}$ Doutor, Prof. titular, Departamento de Engenharia Agrícola UFV/Viçosa - MG, mmauro@ufv.br.

Recebido pelo Conselho Editorial em: 13-9-2010

Aprovado pelo Conselho Editorial em: 15-4-2011
} 
Importantes trabalhos foram dedicados à análise do impacto potencial das mudanças climáticas sobre os recursos hídricos. Tem sido observado que a temperatura média global do ar na superfície aumentou em torno de $0,74{ }^{\circ} \mathrm{C}$ ao longo dos 100 anos, entre 1906 e 2005, e há uma previsão de aumento de 1,1 a $6,4^{\circ} \mathrm{C}$ até 2100 (IPCC, 2007).

A agricultura, por ser fortemente dependente das condições climáticas, provavelmente, sofrerá influência direta das mudanças climáticas na produção agrícola, afetando a vida humana sob muitos aspectos. Como principal responsável pela perda de água nas bacias hidrográficas, a evapotranspiração exerce uma grande influência sobre os processos hidrológicos, sendo que este componente está estreitamente relacionado com a dinâmica da umidade do solo, a recarga dos aquíferos e o escoamento superficial. Consequentemente, a compreensão das variações temporais e espaciais da evapotranspiração é um componente vital nos estudos hidrológicos.

É necessária uma previsão precisa da evapotranspiração da cultura (ETc) para fins de uma irrigação eficiente, para os cálculos hidrológicos do balanço hídrico, planejamento dos recursos hídricos e conhecer o potencial das culturas de sequeiro (DINPASHOH, 2006).

A distribuição e a tendências da evapotranspiração de referência (ETo) são extremamente importantes para planejamento de recursos hídricos e para a agricultura, visto que está sendo amplamente discutido o seu comportamento futuro em função do aquecimento global.

Alguns estudos têm verificado importantes tendências de aumento e, principalmente, diminuição na evaporação medida em tanques e na evapotranspiração de referência (ETo) nas últimas décadas, em diferentes regiões, em todo o mundo. LIANG et al. (2009), analisando a variação anual da ETo na bacia do Rio Taoer, na China, para o período de 1961 a 2005, verificaram um aumento da evapotranspiração de referência da ordem de $0,42 \mathrm{~mm}$ por ano. BANDYOPADHYAY et al. (2009) encontraram certa tendência de decréscimo da evapotranspiração de referência (ETo) para 133 estações meteorológicas localizadas na Índia, mostrando que a diminuição da evapotranspiração foi decorrente do aumento da umidade relativa e da diminuição da velocidade do vento, principalmente.

XU et al. (2006a) investigaram as possíveis causas da diminuição da ETo e quantificaram as contribuições dos principais elementos climáticos na tendência da evapotranspiração, verificando uma redução significativa, a 5\% de probabilidade da evapotranspiração de referência e da evaporação, em tanques para a região da Bacia do Rio Yangtze, na China, no período de 1970 a 2000. Eles observaram que a tendência à diminuição da evapotranspiração pode ser atribuída à tendência estatisticamente significativa de redução da radiação solar. Estes mesmos autores observaram alguma redução significativa, ao nível de 5\% de probabilidade, com tendência de $-1,24$ mm por ano durante 1960-2000, para a ETo anual na bacia do Rio Changjiang, na China (XU et al., 2006b)

No Brasil, poucos estudos têm sido realizados para analisar a variação temporal da evapotranspiração decorrente das novas condições climáticas. Qualquer alteração nos elementos climáticos, em razão do aquecimento global, também afetará a exigência hídrica das culturas.

O objetivo do presente estudo foi identificar as possíveis tendências nas séries climatológicas de temperaturas máxima e mínima, insolação, velocidade do vento e umidade relativa, e analisar os efeitos das mudanças desses elementos climáticos na evapotranspiração de referência e na evapotranspiração da cultura do milho.

\section{MATERIAL E MÉTODOS.}

A área de estudo compreende a região do município de Viçosa, que está localizada na zona da Mata Mineira, na região Sudeste, situada a $20^{\circ} 45^{\prime}$ de latitude sul, $42^{\circ} 50^{\prime}$ de longitude oeste e altitude de $672 \mathrm{~m}$. 
Foram utilizados dados diários dos seguintes elementos do clima: temperatura máxima, temperatura mínima, umidade relativa, velocidade do vento e insolação, coletados na estação climatológica pertencente ao Instituto Nacional de Meteorologia (INMET), no período de 1968 a 2008.

O critério utilizado para o preenchimento de falhas, isto é, ausência de dados necessários ao cálculo da ETo pelo método de PM-FAO, foi o método-padrão recomendado no Boletim da FAO ${ }^{\circ}$ 56 de 1998. Para os meses com dados faltosos, foi considerada a média do mês para o preenchimento da falha.

No estudo das possíveis tendências dos elementos climáticos, foram considerados os valores médios mensais e anuais. Em cada elemento estudado, foram aplicados a análise de regressão e o teste de Mann-Kendall.

\section{Análise de tendência}

O teste de Mann-Kendall e o teste t do coeficiente angular da equação de regressão são os testes mais utilizados para a verificação de presença de tendência em séries de dados (RODERICK \& FARQUHAR, 2005), (WANG et al., 2006) (ZHANG et al., 2010).(XU et al., 2006a).

O teste de Mann-Kendall (MANN, 1945; KENDALL, 1975) é uma análise estatística não paramétrica, que considera a hipótese de estabilidade de uma série temporal. A sucessão de valores ocorre de forma independente, e a distribuição de probabilidade deve permanecer sempre a mesma (série aleatória simples). Considerando uma série temporal de Yi de $n$ termos $(1 \leq \mathrm{i} \leq \mathrm{n})$, a estatística desse teste é dada por:

$$
S=\sum_{i=1}^{n-1} \sum_{j=i+1}^{n} \operatorname{sinal}\left(Y_{j}-Y_{i}\right)
$$

em que,

$$
\operatorname{sinal}\left(Y_{j}-Y_{i}\right)=\left\{\begin{array}{ll}
+1 & \operatorname{se}\left(Y_{j}-Y_{i}\right)>0 \\
0 & \operatorname{se}\left(Y_{j}-Y_{i}\right)=0 \\
-1 & \operatorname{se}\left(Y_{j}-Y_{i}\right)<0
\end{array}\right\}
$$

Para séries com grande número de termos (n), sob a hipótese nula (Ho) de ausência de tendência verdadeira, $\mathrm{S}$ apresenta uma distribuição normal com média zero e variância dada por:

$$
\operatorname{Var}(S)=\frac{n(n-1)(2 n+5)}{18}
$$

Testando a significância estatística de $\mathrm{S}$ para a hipótese alternativa $\left(\mathrm{H}_{1}\right)$, usando um teste bilateral a $\mathrm{H}_{1}$, esta pode ser rejeitada para grandes valores da estatística $\mathrm{z}$, que é dada por:

$$
\mathrm{Z}= \begin{cases}\frac{(\mathrm{S}-1)}{\sqrt{\operatorname{Var}(\mathrm{S})}} & \text { se } \mathrm{S}>0 \\ 0 & \text { se } \mathrm{S}=0 \\ \frac{(\mathrm{S}+1)}{\sqrt{\operatorname{Var}(\mathrm{S})}} & \text { se } \mathrm{S}<0\end{cases}
$$

Com base na análise da estatística z, é feita a decisão final de aceitar ou rejeitar Ho, ou seja, pode-se confirmar a hipótese de estabilidade dos dados ou rejeitá-la a favor da hipótese alternativa, isto é, a de existência de tendência nos dados. O sinal da estatística $z$ indica se a tendência é 
crescente $(z>0)$ ou decrescente $(z<0)$. Em um teste bilateral para tendência, $\mathrm{H}_{1}$ deve ser aceita se $|\mathrm{z}| \leq \mathrm{Z}_{\alpha / 2}$, em que o valor de $\mathrm{Z}_{\alpha / 2}$ é obtido da tabela na normal-padrão.

A análise de regressão é utilizada para identificar a tendência na série temporal por meio do teste de significância do coeficiente angular da reta:

$$
\mathrm{Y}=a+b \mathrm{X}
$$

em que,

$\mathrm{Y}=$ elemento do clima;

$\mathrm{X}=$ tempo, $\mathrm{e}$

$a$ e $b=$ coeficientes da regressão calculados pelo método dos mínimos quadrados.

Considerando a regressão linear de Y com variável aleatória no tempo X, a hipótese nula Ho de que não existe uma tendência, é testada por meio do teste $\mathrm{t}$ de Student com n-2 graus de liberdade:

$$
\mathrm{t}=\frac{\mathrm{r} \sqrt{\mathrm{n}-2}}{\sqrt{1-\mathrm{r}^{2}}}=\frac{b}{s / \sqrt{S S x}}
$$

em que,

$\mathrm{n}=$ tamanho da amostra;

$\mathrm{r}=$ coeficiente de correlação de Pearson;

$\mathrm{s}=$ desvio-padrão dos resíduos;

$b=$ coeficiente angular da reta, e

$\mathrm{SS}_{\mathrm{X}}=$ soma dos quadrados da variável independente (tempo em análise de tendência).

A hipótese de que não há tendência, é rejeitada quando o valor de t calculado pela equação 5 é maior, em valor absoluto, do que o valor crítico t $\alpha / 2$, n-2, tabelado, a um determinado nível de significância $\alpha$. Para validar a hipótese de que o coeficiente angular da regressão é significativo, foi feito o teste F para a regressão.

Para a aplicação dos testes estatísticos para a verificação da presença de tendência nas séries de dados, foi considerado que os dados são independentes e identicamente distribuídos.

\section{Cálculo da evapotranspiração}

Com o objetivo de padronizar o conceito de evapotranspiração em diferentes regiões, a FAO (Food and Agriculture Organization) propôs a evapotranspiração de referência (ETo), definida como a taxa de evapotranspiração de uma superfície de referência com altura de $0,12 \mathrm{~m}$, uma

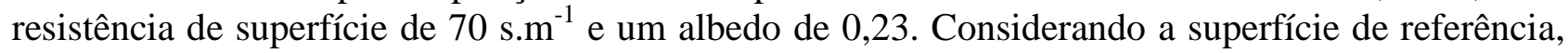
uma cultura hipotética com altura uniforme, sem restrições de água, crescendo ativamente e sombreando completamente o solo, o método de Penman-Monteith (PM-FAO) foi o método recomendado pela FAO como padrão para calcular ETo e tem sido utilizado em todo o mundo (ALLEN et al., 1998).

Os dados meteorológicos diários utilizados para a obtenção da evapotranspiração de referência diária foram: temperatura máxima, temperatura mínima, umidade relativa, velocidade do vento e insolação. Para o cálculo da evapotranspiração e verificação dos possíveis efeitos das alterações dos elementos climáticos na evapotranspiração, utilizou-se a equação de Penman-Monteith (FAO), parametrizada por ALLEN et al. (1998), para intervalo de tempo de 24 horas.

$$
E T_{0}=\frac{0,408 \Delta\left(R_{\mathrm{n}}-G\right)+\gamma \frac{900}{T+273} u_{2}(e s-e a)}{\Delta+\gamma\left(1+0,34 u_{2}\right)}
$$


em que,

ETo = evapotranspiração de referência $(\mathrm{mm} /$ dia $)$;

$\mathrm{Rn}=$ saldo de radiação à superfície da cultura $\left(\mathrm{MJ} \mathrm{m}^{-2}\right)$;

$\mathrm{G}=$ densidade do fluxo de calor do solo $\left(\mathrm{MJ} \mathrm{m}^{-2}\right)$;

$\mathrm{T}=$ temperatura do ar a $2 \mathrm{~m}$ de altura $\left({ }^{\circ} \mathrm{C}\right)$;

$\mathrm{u}_{2}=$ velocidade de vento a $2 \mathrm{~m}$ de altura $\left(\mathrm{m} \mathrm{s}^{-1}\right)$;

es $=$ pressão de vapor de saturação média diária $(\mathrm{kPa})$;

ea $=$ pressão parcial de vapor média diária $(\mathrm{kPa})$;

$\Delta=$ declividade da curva de pressão de vapor de saturação $\left(\mathrm{kPa}^{\circ} \mathrm{C}^{-1}\right)$, e

$\gamma=$ coeficiente psicrométrico $\left(\mathrm{kPa}^{\circ} \mathrm{C}^{-1}\right)$.

As equações padronizadas para o cálculo de todos os parâmetros da equação (6) são apresentadas em ALLEN et al. (1998).

\section{Cálculo do coeficiente dual de cultura}

A época de plantio da cultura do milho, utilizada na simulação, foi em primeiro de outubro para o milho de safra normal e primeiro de fevereiro para o milho safrinha, sendo que o ciclo da cultura considerado foi de 125 e 135 dias, respectivamente.

O coeficiente de cultura $(\mathrm{Kc})$ é basicamente a razão entre a evapotranspiração da cultura (ETc) e a de referência (ETo), e representa uma integração dos efeitos das principais características que distinguem a cultura em questão do padrão grama (referência). Estas características são a altura da cultura (que afeta a rugosidade e a resistência aerodinâmica); resistência da cultura (afetada pela área foliar, a fração de solo coberto pela vegetação, idade da folha e condição de umidade na superfície do solo) e o albedo da cultura (afetado pela fração do solo coberto pela vegetação e pela umidade na superfície do solo).

Assim, para o cálculo da evapotranspiração da cultura, utiliza-se a equação:

$$
\mathrm{ETc}=\mathrm{ETo} \times \mathrm{Kc}
$$

em que, Kc é o coeficiente dual de cultura, para os diferentes estádios de crescimento.

Para a obtenção do coeficiente dual de cultura, aplicou-se o método proposto por ALLEN et al. (2006), no qual o coeficiente de cultura Kc pode ser decomposto em dois coeficientes, um para a transpiração das plantas - o coeficiente basal da cultura $(\mathrm{Kcb})$ - e outro para a evaporação da água do solo $(\mathrm{Ke})$.

O coeficiente basal da cultura $(\mathrm{Kcb})$ refere-se à relação entre a evapotranspiração da cultura e a evapotranspiração de referência, quando apenas a superfície do solo está aparentemente seca, mas a transpiração está ocorrendo em uma taxa potencial, ou seja, a água no sistema radicular não é fator limitante para a transpiração.

A curva de Kcb é dividida em quatro estádios de crescimento: inicial, de desenvolvimento, intermediário e final. Os estádios inicial e intermediário são caracterizados por segmentos de linha horizontal e os estádios de desenvolvimento e final do ciclo são caracterizados por aumento e queda do coeficiente de cultura, respectivamente. Os valores indicativos de Kcb são aqueles tabelados em ALLEN et al.(1998), sendo que são necessários apenas três valores para descrever e construir a curva do coeficiente de cultura. Depois de selecionar e ajustar os valores Kcb correspondentes aos estádios inicial $\left(\mathrm{Kcb}_{\text {ini }}\right)$, intermediário $\left(\mathrm{Kcb}_{\text {médio }}\right)$ e final $\left(\mathrm{Kcb}_{\text {final }}\right)$, a curva do coeficiente de cultura é elaborada, e os coeficientes Kcb são obtidos desta.

No caso das condições de umidade relativa mínima e da velocidade do vento média serem diferentes de $45 \%$ e de $2 \mathrm{~m} / \mathrm{s}$, respectivamente, faz-se a seguinte correção para os coeficientes de cultura do estádio intermediário e final: 


$$
\mathrm{Kcb}=\mathrm{Kcb}_{\text {(tab) }}+\left[0,04\left(U_{2}-2\right)-0,004\left(R H_{\text {min }}-45\right)\right]\left(\frac{h}{3}\right)^{0,3}
$$

em que,

$\mathrm{h}$ é a altura média das plantas $(\mathrm{m})$;

$\mathrm{U}_{2}$ é a velocidade média do vento durante o período, determinada ou corrigida para $2 \mathrm{~m}$ de altura $(\mathrm{m} / \mathrm{s})$;

$\mathrm{RH}_{\min }$ é a média da umidade relativa mínima diária para o período (\%).

O coeficiente de evaporação da água do solo, Ke, descreve o componente de evaporação da ETc. Quando o solo está molhado, depois da chuva ou irrigação, Ke é máximo. Sempre que a superfície do solo está seca, Ke é pequeno ou até mesmo zero quando não há água, próxima à superfície do solo para a evaporação.

Quando o solo está molhado, a evaporação ocorre em sua taxa máxima. No entanto, o coeficiente de cultura $(\mathrm{Kc}=\mathrm{Kcb}+\mathrm{Ke})$ nunca poderá exceder a um valor máximo.

Este valor máximo é determinado pela energia disponível na superfície do solo para a evaporação $\left(\mathrm{Kcb}+\mathrm{K}_{\mathrm{e}} \leq \mathrm{Kc}_{\max }\right)$, ou $\mathrm{K}_{\mathrm{e}} \leq\left(\mathrm{Kc}_{\max }-\mathrm{Kcb}\right)$. $\mathrm{O}$ valor de $\mathrm{Kc}_{\max }$ representa um limite máximo para a evaporação e a transpiração de toda a superfície cultivada e é introduzido para refletir as restrições naturais, definidas pela energia disponível. $\mathrm{O}$ valor do $\mathrm{Kc}_{\max }$ é fornecido pela seguinte equação.

$$
\mathrm{Kc}_{\text {max }}=\max \left(\left\{1,2+\left[0,04\left(U_{2}-2\right)-0,004\left(R H_{\text {min }}-45\right)\right]\left(\frac{h}{3}\right)^{0,3}\right\},\{\mathrm{Kcb}+0,05\}\right)
$$

Ao longo do tempo, depois de uma chuva ou irrigação, o solo começa a secar, e a quantidade de evaporação reduz-se proporcionalmente à quantidade de água existente na superfície do solo, então:

$$
\mathrm{Ke}=\mathrm{Kr}\left(\mathrm{Kc}_{\max }-\mathrm{Kcb}\right) \leq \mathrm{f}_{\mathrm{ew}} \mathrm{Kc}_{\max }
$$

em que,

Kr é o coeficiente de redução da evaporação, dependente da quantidade de água armazenada na camada de solo, e

$\mathrm{f}_{\text {ew }}$, a fração molhada do solo exposta à radiação solar.

O coeficiente de redução de evaporação apresenta dois estádios, um estádio de limitação de energia (estádio 1) e outro de redução da evaporação (estádio 2).

Durante o estádio 1, o solo permanece úmido e presume-se que a evaporação irá ocorrer a uma taxa máxima, limitada apenas pela disponibilidade de energia na superfície do solo e, portanto, $\mathrm{K}_{\mathrm{r}}=1$. No final do estádio 1 , depois da diminuição da água no solo, a depleção da água no solo é igual ao REW (água prontamente disponível).

Quando o teor de água na camada superior do solo se torna limitante, $\mathrm{Kr}$ diminui, podendo torna-se zero. $\mathrm{O} \mathrm{Kr}$ é determinado pelo balanço hídrico diário da camada superior do solo. Para condições limitantes de água (estádio 2), a determinação do coeficiente de redução da evapotranspiração é dada pela equação 11.

$$
\mathrm{Kr}=\frac{\mathrm{TEW}-\mathrm{Dr}_{\mathrm{i}-1}}{\mathrm{TEW}-\mathrm{REW}}
$$

em que,

TEW = lâmina máxima de água que pode ser evaporada da superfície do solo, quando este estiver completamente molhado (mm); 
$\mathrm{D}_{\mathrm{r}, \mathrm{i}-1}=$ lâmina acumulada de evaporação (depleção) da camada superficial do solo, no final do dia i-1 (dia anterior) ( $\mathrm{mm})$, e

REW = lâmina acumulada de evaporação (depleção), no fim do estágio 1 (mm).

Para os cálculos, foram considerados os valores de Kcb inicial, intermediário e final de 0,15 ; 1,15 e 0,15 , respectivamente, conforme recomendações de ALLEN et al. (2006).

\section{RESULTADOS E DISCUSSÃO}

Na Tabela 1, têm-se os resultados das análises de tendência para as séries estudadas. Pode-se notar um aumento estatisticamente significativo da temperatura máxima média para os meses de abril, agosto, setembro, outubro e para a temperatura máxima média anual. Todavia, apenas o mês de janeiro apresentou uma diminuição da temperatura máxima, porém esta diminuição não foi estatisticamente significativa, a $5 \%$ de probabilidade, pelo teste de $\mathrm{t}$ de Student e pelo teste de Mann-Kendall.

Pelos testes de tendência, apenas o mês de agosto não apresentou uma tendência significativa de aumento na temperatura mínima média mensal. Além disso, pode ser verificado um aumento da temperatura mínima em todos os meses do ano e, consequentemente, na temperatura mínima média.

Nota-se que houve um aumento significativo da nebulosidade nos meses de janeiro, maio e dezembro, acarretando, assim, uma redução do número de horas de brilho solar. Foi verificado, também, redução da insolação, para os outros meses do ano, além de janeiro, maio e dezembro, porém esta diminuição não foi estatisticamente significativa para esses meses. Apenas o mês de outubro apresenta um aumento da insolação, porém não significativo a $5 \%$ de probabilidade, pelo teste t e de Mann-Kendall. No entanto, a redução ao longo dos meses do ano reflete uma redução significativa da insolação média anual.

TABELA 1. Análise de tendência para os elementos do clima estudados, em Viçosa-MG (de 1968 a 2008). Trend analysis for the climate elements studied in Viçosa, MG (from 1968 to 2008).

\begin{tabular}{cccccc}
\hline MESES & $\begin{array}{c}\text { Temperatura } \\
\text { máxima }\end{array}$ & $\begin{array}{c}\text { Temperatura } \\
\text { mínima }\end{array}$ & $\begin{array}{c}\text { Insolação } \\
\text { Velocidade do } \\
\text { vento }\end{array}$ & $\begin{array}{c}\text { Umidade } \\
\text { relativa }\end{array}$ \\
\hline Janeiro & - & $\uparrow$ & $\downarrow$ & $\downarrow$ & - \\
Fevereiro & - & $\uparrow$ & - & $\downarrow$ & - \\
Março & - & $\uparrow$ & - & $\downarrow$ & - \\
Abril & $\uparrow$ & $\uparrow$ & - & $\downarrow$ & - \\
Maio & - & $\uparrow$ & $\downarrow$ & $\downarrow$ & - \\
Junho & - & $\uparrow$ & - & $\downarrow$ & - \\
Julho & - & $\uparrow$ & - & $\downarrow$ & - \\
Agosto & $\uparrow$ & - & - & $\downarrow$ & - \\
Setembro & $\uparrow$ & $\uparrow$ & - & $\downarrow$ & $\downarrow$ \\
Outubro & $\uparrow$ & $\uparrow$ & - & $\downarrow$ & - \\
Novembro & - & $\uparrow$ & - & $\downarrow$ & - \\
Dezembro & - & $\uparrow$ & $\downarrow$ & $\downarrow$ & - \\
Anual & $\uparrow$ & $\uparrow$ & $\downarrow$ & $\downarrow$ & $\downarrow$ \\
\hline
\end{tabular}

$\uparrow$ tendência de aumento (significativo a 5\%) $\downarrow$ tendência a diminuição (significativo a 5\%).

A velocidade do vento teve um comportamento atípico em relação às outras variáveis estudadas, apresentando fortes reduções ao longo da série e em todos os meses do ano. 
A umidade relativa apresentou tendência a diminuição apenas para o mês de outubro, sendo que, para os outros meses, não apresentou nenhuma tendência.

\section{Análise da evapotranspiração}

A partir dos dados de evapotranspiração de referência diária, foi calculada a evapotranspiração mensal e anual e, assim, foi analisado o comportamento da evapotranspiração de referência (ETo) ao longo dos anos.

Na Figura 1, são mostrados os valores de evapotranspiração média para os meses do ano. $\mathrm{O}$ mês de junho apresenta a menor demanda evapotranspirativa, apresentando uma evapotranspiração média de $60 \mathrm{~mm}$ por mês. O menor valor encontrado para este mês foi de $49 \mathrm{~mm}$ no ano de 1978 . Esta demanda, no mês de junho-se deve à baixa temperatura encontrada na região e à menor radiação incidente nesta época do ano. A maior demanda evapotranspirativa é observada no mês de janeiro, que apresenta uma evapotranspiração média de $125 \mathrm{~mm}$ por mês.

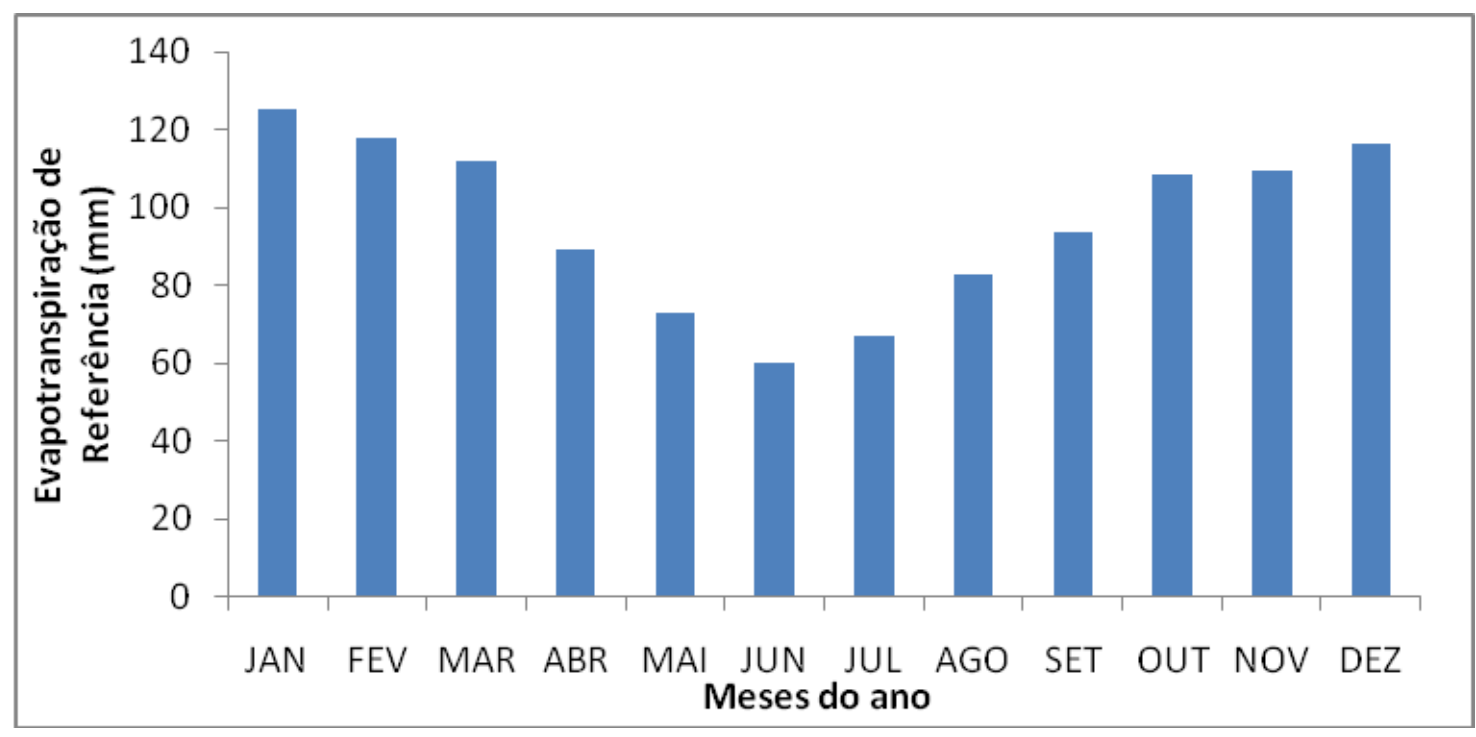

FIGURA 1. Evapotranspiração de referência média mensal, em Viçosa--MG (médias de 1968 a 2008). Monthly average of reference evapotranspiration in Viçosa, MG (mean from 1968 to 2008).

$\mathrm{Na}$ Tabela 2, encontram-se os resultados dos testes de tendência para a série de dados de evapotranspiração de referência.

Analisando a equação de regressão da evapotranspiração de referência com o tempo, a maioria dos meses analisados apresentou uma diminuição significativa da ETo, a 5\% de probabilidade, sendo que somente o mês de outubro não apresentou tendência à diminuição. Apesar da diminuição observada nos meses de abril e setembro, esta redução não foi estatisticamente significativa. A tendência da ETo é função da tendência observada para os elementos climáticos.

Nota-se que apenas os meses que não apresentaram tendência significativa pelo teste $t$ do coeficiente angular, foram os meses que não mostraram resultados significativos pelo teste $\mathrm{F}$.

Os resultados do teste $\mathrm{t}$ e do teste de Mann-Kendall apresentaram boa concordância entre si, apenas o mês de dezembro apresentou uma tendência a diminuição estatisticamente significativa, a $1 \%$ de probabilidade, pelo teste t. Porém, não foi constatada esta tendência pelo teste de MannKendall. 
TABELA 2. Equação de regressão linear, coeficiente de determinação, teste F e teste de MannKendall (TMK) para a evapotranspiração de referência, em Viçosa-MG (de 1968 a 2008). Linear regression equation, coefficient of determination, $F$ test and MannKendall test (TMK) for reference evapotranspiration in Viçosa, MG (from 1968 to 2008).

\begin{tabular}{ccccc}
\hline Mês & $\begin{array}{c}\text { Regressão Linear } \\
(\mathrm{y}=\text { ETo e } \mathrm{x}=\text { ano })\end{array}$ & $\mathrm{R}^{2}$ & $\begin{array}{c}\text { Teste } \mathrm{F} \\
\text { (significância) }\end{array}$ & TMK \\
\hline Janeiro & $\mathrm{y}=-0,728 \mathrm{x}+1573^{*}$ & 0,15 & 0.0127 & $-2.21^{*}$ \\
Fevereiro & $\mathrm{y}=-0,537 \mathrm{x}+1186^{* *}$ & 0,16 & 0.0098 & $-2.21^{*}$ \\
Março & $\mathrm{y}=-0,488 \mathrm{x}+1084^{* *}$ & 0,19 & 0.0047 & $-2.71^{* *}$ \\
Abril & $\mathrm{y}=-0,177 \mathrm{x}+441,3$ & 0,05 & 0.1746 & -0.03 \\
Maio & $\mathrm{y}=-0,388 \mathrm{x}+844,5 * *$ & 0,30 & 0.0002 & $-2.74 * *$ \\
Junho & $\mathrm{y}=-0,315 \mathrm{x}+686,5 * *$ & 0,27 & 0.0005 & $-2.44^{*}$ \\
Julho & $\mathrm{y}=-0,300 \mathrm{x}+665,1 * *$ & 0,29 & 0.0003 & $-3.02^{* *}$ \\
Agosto & $\mathrm{y}=-0,347 \mathrm{x}+773,3 * *$ & 0,20 & 0.0034 & $-2.30^{*}$ \\
Setembro & $\mathrm{y}=-0,246 \mathrm{x}+582,9$ & 0,15 & 0.1435 & -1.16 \\
Outubro & $\mathrm{y}=0,053 \mathrm{x}+2,194$ & 0,10 & 0.7307 & 0.80 \\
Novembro & $\mathrm{y}=-0,342 \mathrm{x}+789,1^{*}$ & 0,10 & 0.0489 & $-1.97 *$ \\
Dezembro & $\mathrm{y}=-0,467 \mathrm{x}+1046^{* *}$ & 0,17 & 0.0079 & -1.90 \\
Anual & $\mathrm{y}=-4.285 \mathrm{x}+9674 * *$ & 0,28 & 0.0004 & $-2.98^{* *}$ \\
\hline
\end{tabular}

*significativo a $5 \%$ de probabilidade, ** significativo a $1 \%$ de probabilidade.

Os decréscimos mais acentuados foram da ordem de 0,72;0,53 e 0,48 mm/mês, por ano, para os meses de janeiro, fevereiro e março, respectivamente. Para esses meses, a maior diminuição da ETo deve-se principalmente à maior redução da insolação, aliado ao aumento significativo da temperatura mínima e ao pequeno aumento da temperatura máxima, sendo que este aumento desproporcional das temperaturas reduz o déficit de pressão de vapor. Outro fator importante é a redução da velocidade do vento que foi verificada para todos os meses.

Os meses de abril, junho, julho, agosto, setembro e novembro apresentaram decréscimos inferiores aos outros meses anteriormente citados. A menor diminuição da ETo para esses meses, em comparação aos outros, deve-se a uma pequena diminuição da insolação verificada para esses meses.

O mês de outubro foi o único a não apresentar uma tendência à diminuição da evapotranspiração de referência, em função do balanceamento das variáveis meteorológicas na equação de Penman-Monteith (FAO). Foi verificado que apenas o mês de outubro apresentou tendência estatisticamente significativa à diminuição da umidade relativa. Também ocorreu aumento da temperatura máxima, ocasionando o comportamento contrário aos outros meses estudados.

A evapotranspiração de referência anual é o reflexo da evapotranspiração de todos os meses. Assim, a redução da ETo, ao longo dos meses, proporcionou um decréscimo, da ordem de 4,2 mm por ano (Figura 2). Considerando este valor ao longo dos 41 anos estudados, ocorreu uma redução de, aproximadamente, $172 \mathrm{~mm}$ na demanda evapotranspirativa, o que representaria uma diminuição de $15 \%$ em relação à média. 


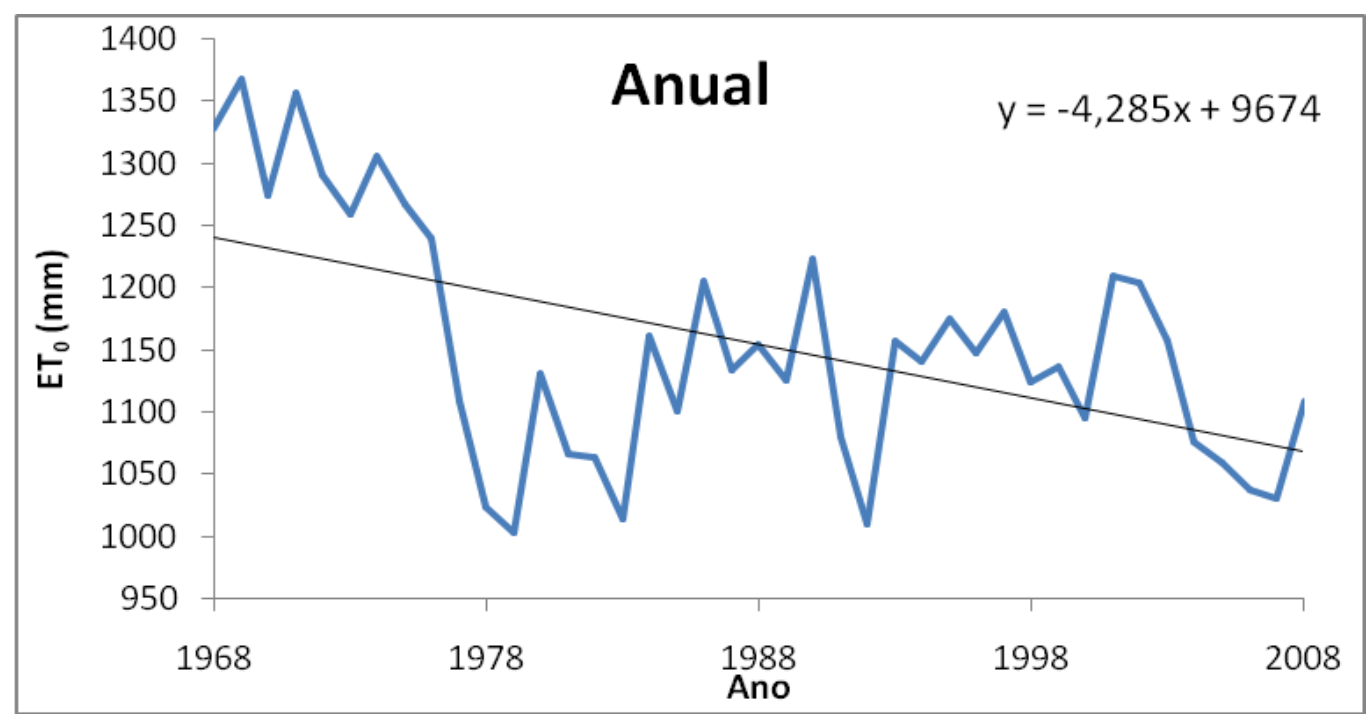

FIGURA 2. Evapotranspiração de referência anual, em Viçosa-MG (de 1968 a 2008). Annual reference evapotranspiration in Viçosa, MG (from 1968 to 2008).

A tendência à diminuição da evapotranspiração anual foi causada, principalmente, pela diminuição da insolação, da velocidade do vento e pelo aumento da temperatura mínima superior à temperatura máxima.

\section{Evapotranspiração da cultura}

A evapotranspiração da cultura (ETc) foi obtida por meio da multiplicação da ETo pelo coeficiente dual de cultura em base diária. Desta maneira, realizou-se o somatório da ETc diária, obtendo-se, assim, a evapotranspiração do ciclo da cultura para o milho safrinha e o de safra normal.

Nas Figuras 3 e 4, encontram-se os gráficos da distribuição da ETo e da ETc para o ciclo da cultura, considerando o milho de safra normal e safrinha, respectivamente, ao longo dos 41 anos analisados. Os asteriscos nas equações de regressão linear indicam que a diminuição da evapotranspiração da cultura é estatisticamente significativa, pelo teste t de Student.
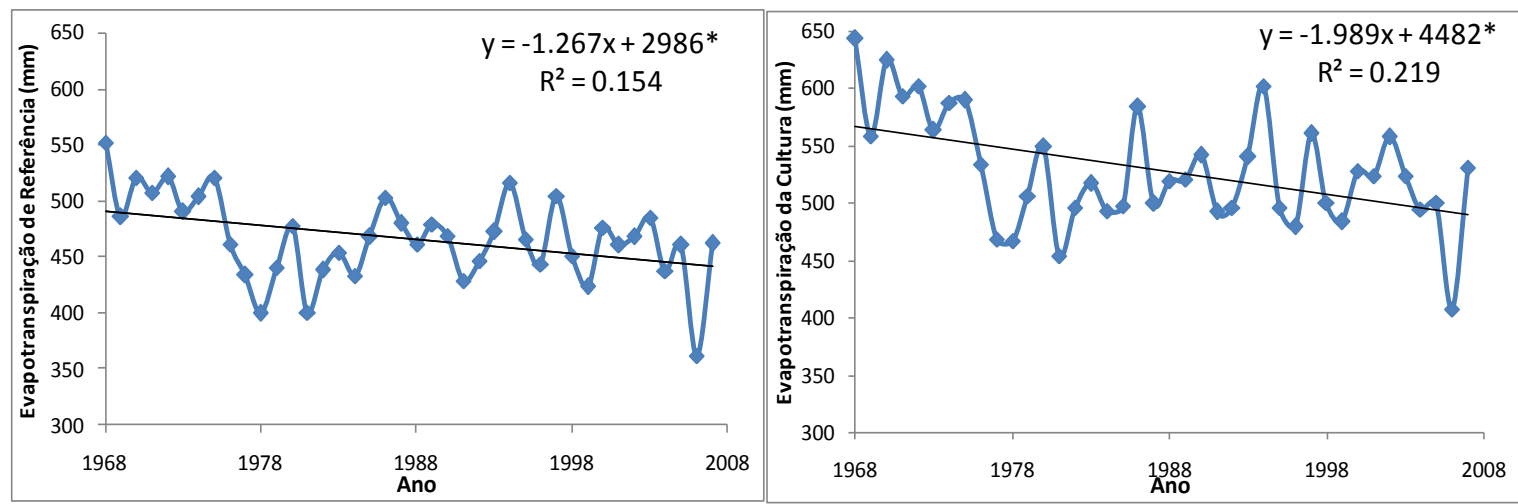

FIGURA 3. Evapotranspiração de referência e da cultura do milho para o período de outubro a fevereiro em Viçosa-MG. Reference and maize evapotranspiration for the period from October to February in Viçosa, MG.

A cultura do milho, de safra normal, apresentou uma demanda evapotranspirativa média de $529 \mathrm{~mm}$ por ciclo da cultura, sendo que, para este período, a ETo média do ciclo apresentou um valor médio de $466 \mathrm{~mm}$. Para o milho safrinha, foi observada uma demanda evapotranspirativa 
média de 478, mm e a ETo média para o ciclo da cultura foi de $421 \mathrm{~mm}$, sendo que a duração do ciclo do milho safrinha foi de 135 dias.

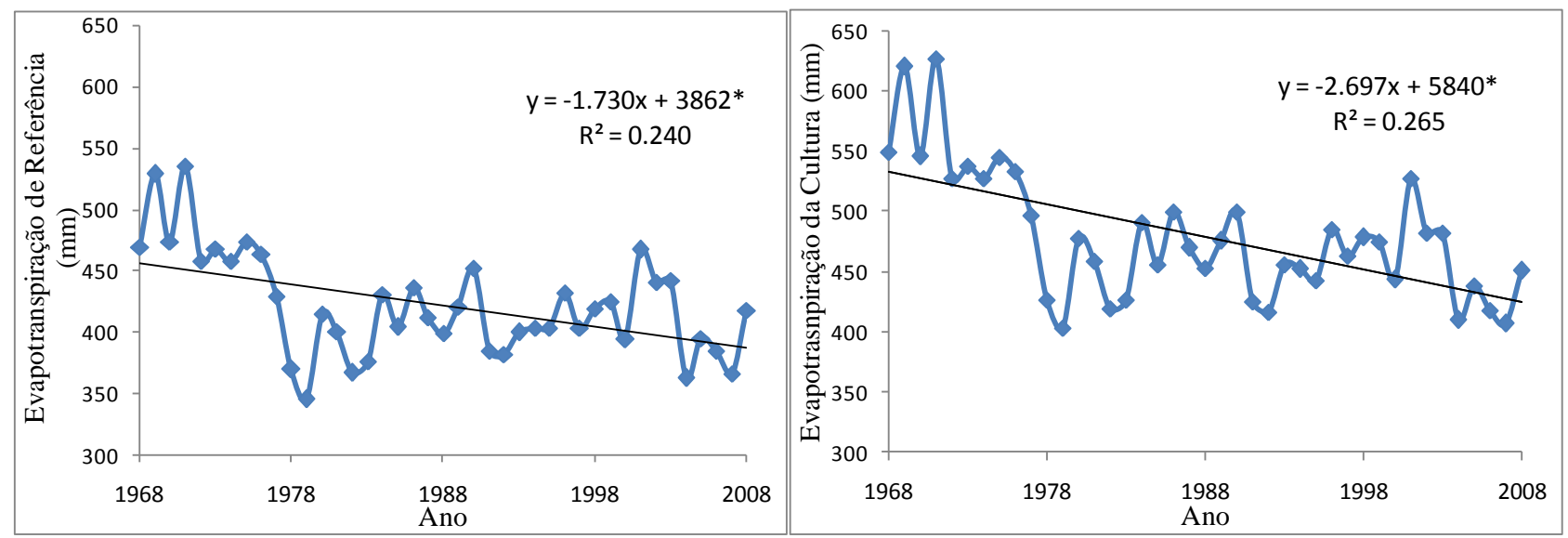

FIGURA 4. Evapotranspiração de referência e da cultura do milho para o período de fevereiro a junho, em Viçosa-MG. Reference and maize evapotranspiration for the period from February to June in Viçosa, MG.

Como a ETc reflete as variações da ETo e das características da cultura (Kc), assim, a diminuição da evapotranspiração de referência observada, ao longo dos meses do ano, influenciou diretamente na ETc, mostrando os meses de aumento e diminuição da mesma forma que a ETo, para os meses do ano.

Ao contrário do que se esperava, verificou-se que as declividades da reta da equação de regressão da ETo e da ETc apresentaram valores diferentes. Para a safra normal, a diminuição da ETc foi da ordem de 1,98 $\mathrm{mm}$ por safra/ano e da ETo foi de 1,26 $\mathrm{mm}$ por safra/ano. Este mesmo comportamento foi verificado para o milho safrinha, que apresentou uma redução da ETc e da ETo de 2,69 e $1,73 \mathrm{~mm} / \mathrm{safra}$ por ano, respectivamente.

Esta diferença ocorre porque o coeficiente de cultura dual não apresenta valores fixos para cada estádio de desenvolvimento da cultura, como ocorre com o coeficiente de cultura único (Kc único). Portanto, o coeficiente de cultura dual varia com as condições de umidade do solo. Desta maneira, a precipitação influencia no coeficiente de cultura dual. Por exemplo, considerando uma situação em que se tem alta demanda evapotranspirativa e se, no período em análise, ocorre grande quantidade de chuvas, o coeficiente de cultura dual tende a ser maior do que numa mesma situação com menor quantidade de chuvas. Isso ocorre porque o coeficiente de cultura dual leva em consideração o componente de evaporação da água no solo $(\mathrm{Ke})$. Quando o solo está molhado, a evapotranspiração ocorre em sua taxa máxima, ou seja, o coeficiente de cultura dual também será máximo. Por isso, foi encontrado comportamento diferente nas reduções da ETc e da ETo.

As reduções da ETc apresentam valores bem próximos, porém a menor redução da evapotranspiração, para o milho safra normal, ocorre porque, no período deste ciclo, o mês de outubro apresentou um pequeno aumento da ETo, que contribuiu para a menor diminuição da ETc. Para o milho safrinha, houve uma redução da ETo em todos os meses do ciclo da cultura.

\section{CONCLUSÕES}

Diante das tendências analisadas para as séries de dados, foi verificada uma diminuição na evapotranspiração de referência para todos os meses do ano, exceto o mês de outubro; consequentemente, a evapotranspiração de referência apresentou diminuição de 4,2 mm por ano. Seguindo este padrão, a evapotranspiração da cultura do milho apresentou redução da ordem de 1,98 e 2,69 mm por ano, para o milho de safra normal e o milho safrinha, respectivamente. 


\section{AGRADECIMENTOS}

Ao $\mathrm{CNPq}$, pelo suporte financeiro que permitiu o desenvolvimento deste trabalho de pesquisa, e ao INMET, pelo fornecimento dos dados.

\section{REFERÊNCIAS}

ALLEN, R. G.; PEREIRA, L. S.; RAES, D.; SMITH, M. Crop evapotranspiration: guidelines for computing crop water requirements. Rome: FAO, 1998. 300 p. (Irrigation and Drainage Paper, 56).

ALLEN, R.G.; PEREIRA, L.S.; RAES, D.; SMITH, J. Evapotranspiration del cultivo: guias para la determinación de los requerimientos de agua de los cultivos. Roma: FAO, 2006. 298 p. (Estudio Riego e Drenaje Paper, 56).

BANDYOPADHYAY, A.; BHADRA, N. S.; RAGHUWANSHI, R.; SINGH, J. Temporal Trends in Estimates of Reference Evapotranspiration over India. Journal of Hydrologic Engineering, Reston, v.14, p.508-515, 2009.

DINPASHOH, A.Y. Study of reference crop evapotranspiration in I.R. of Iran. Agricultural Water Management, Amsterdam, n. 84. p. 123-129., 2006.

IPCC. Climate Change 2007: The physical science basis - Summary for Policy Makers.

Contribution of working group I to the Fourth Assessment Report of the Intergovernmental Panel on Climate Change, 2007. 21p.

KENDALL, M. G. Rank correlation methods. London: Charles Griffin, 1975.

LIANG, L.; LI, L.; LIU, Q. Temporal variation of reference evapotranspiration during 1961-2005 in the Taoer River basin of Northeast China. Agricultural and Forest Meteorology, Amsterdam, v.150. n.2. p. 298-306, 2009.

MANN, H. B. Non-parametric test against trend. Econometrika, Princeton, v. 13, p. 245-259, 1945.

RODERICK, M.L.; FARQUHAR, G.D. Changes in New Zealand pan evaporation since 1970s. International Journal of Climatology, Chichester, v. 25, p. 2.031-2.039, 2005.

WANG, Y.; JIANG, T.; BOTHE, O.; FRAEDRICH, K. Changes of pan evaporation and reference evapotranspiration in the Yangtze River basin. Theoretical and Applied Climatology, Wien, v. 90, p. 13-23, 2006.

XU, C.Y.; GONG, L.; JIANG, T.; CHEN, D. Decreasing reference evapotranspiration in a warming climate - a case of Changjiang (Yangtze River) catchment during 1970-2000. Advances in Atmospheric Sciences, Beiying, v. 23, n. 4, p. 513-520, 2006 a.

XU, C.Y.; GONG, L.; JIANG, T.; CHEN, D.; SINGH, V.P. Analysis of spatial distribution and temporal trend of reference evapotranspiration in Changjiang (Yangtze River) basin. Journal of Hydrology, Amsterdam, v. 30. n.1-2. p. 81-93, 2006 b.

YU, P. S.; TAO, C. Y.; CHOU, C. C. Effects of Climate Change on evapotranspiration from Paddy Fields in Southern Taiwan, Climatic Change, Dordrecht, v. 54, n. 1-2, p. 165-179, jul. 2002.

ZHANG, Q.;XU, C-Y.; TAO, H.;JIANG, T.; CHEN, Y. Climate changes and their impacts on water resources in the arid regions: a case study of the Tarim River basin, China. Stochastic Environmental Research and Risk Assessment, Berlin, v. 24. p. 349-358, 2010. 